\title{
Serum matrix metalloproteinase- 9 is a valuable biomarker for identification of abdominal and thoracic aortic aneurysm: a case-control study
}

\author{
Tan Li ${ }^{1}$, Bo Jiang ${ }^{2}$, Xuan Li ${ }^{2}$, Hai-yang Sun ${ }^{1}$, Xin-tong Li $i^{2}$, Jing-jing Jing ${ }^{3}$ and Jun Yang ${ }^{1 *}$ (i)
}

\begin{abstract}
Background: Matrix metalloproteinase-9 (MMP9) has been reported to play a key role in the pathogenesis of aortic aneurysm. However, few studies have assessed serum MMP9 levels in both abdominal aortic aneurysm (AAA) and thoracic aortic aneurysm (TAA). In this study, we investigated the serum levels of MMP9 in aortic aneurysm to evaluate its predictive and diagnostic efficacy for AAA and TAA, and explored the association of MMP9 with circulating laboratory markers.

Methods: A total of 296 subjects were enrolled, including 105 AAA patients, 79 TAA patients and 112 healthy controls. The levels of serum MMP9 were detected by enzyme-linked immunosorbent assay (ELISA).

Results: Compared to control group, both AAA and TAA patients had higher serum MMP9 levels in the overall comparison and subgroup analysis based on subjects aged $<65$ years, either male or female, hypertension, nondiabetes and non-hyperlipidemia (all $P<0.05$ ). Moreover, MMP9 levels were significantly higher in TAA group than those in AAA group in the total comparison, and this discrepancy was also found in the non-diabetes, nonhyperlipidemia and aortic diameter $\geq 5.5 \mathrm{~cm}$ subgroup analysis. Serum MMP9 levels were influenced by age and hypertension. There was a positive association of serum MMP9 with CRP $(r=0.33, P<0.001)$ and Hcy $(r=0.199$, $P=0.033$ ). Multiple logistic analyses showed that serum MMP9 was an independent risk factor for AAA and TAA. Based on receiver operating characteristic (ROC) analysis, the area under the curve (AUC) of MMP9 for predicting TAA was 0.83 with $70 \%$ sensitivity and $91 \%$ specificity, while the AUC of MMP9 to detect AAA was 0.69 and the sensitivity and specificity were $50 \%$ and $88 \%$.
\end{abstract}

Conclusions: Serum MMP9 was closely related to the existence of aortic aneurysms and could be a valuable marker for the discrimination of aortic aneurysm, especially for TAA.

Keywords: Matrix metalloproteinase-9, Abdominal aortic aneurysm, Thoracic aortic aneurysm, C- reactive protein

\section{Background}

Aortic aneurysm is a complex and dangerous vascular disease that results from the multifactorial interaction of genetic and environmental factors [1]. According to anatomical locations, aortic aneurysm is generally divided into abdominal aortic aneurysm (AAA) and thoracic

\footnotetext{
* Correspondence: yangjun@cmu1h.com

${ }^{1}$ Department of Cardiovascular Ultrasound, the First Hospital of China Medical University, No.155 Nanjing Bei Street, Heping District, Shenyang 110001, China

Full list of author information is available at the end of the article
}

aortic aneurysm (TAA). They may share some similarities in pathogenesis and histological phenotypes that both involve the metabolic imbalance and progressive weakening of aortic wall [2, 3], but not for syndrome and bicuspid valve associated TAA. Matrix metalloproteinase-9 (MMP9) is a gelatinase with proteolytic activity on extracellular matrix degradation in aortic wall and its excessive production can lead to progressive aortic remodeling and dilatation [2]. Any cause increasing the activity of aortic endothelial cells, smooth muscle cells and infiltrating

(c) The Author(s). 2018 Open Access This article is distributed under the terms of the Creative Commons Attribution 4.0 International License (http://creativecommons.org/licenses/by/4.0/), which permits unrestricted use, distribution, and 
inflammatory cells can produce a large amount of MMP9 released into blood circulation [4].

Evidence has demonstrated higher circulating MMP9 levels in AAA patients [5, 6]. As for TAA, studies often focused on the gene variation and tissue expression of MMP9 [7, 8], but much less is known about the association between serum MMP9 and TAA. A study by Meffert et al. suggested no different expression of serum MMP9 between TAA with hyperlipidemia and without hyperlipidemia [9]. Based on small sample sizes, Tsarouhas et al. revealed that there were increased MMP9 levels in TAA serum and tissue [10], however, Karapanagiotidis et al. showed that TAA patients had lower serum MMP9 levels [11]. To our knowledge, there were no studies available on detailed comparison of serum MMP9 levels in AAA and TAA, and the performance of serum MMP9 for identification of aortic aneurysm is still unknown. To date, circulating biomarkers, such as C-reactive protein (CRP), homocysteine (Hcy) and Cystatin C (Cys-c), have been analyzed in aortic aneurysmal diseases, but it remains unclear whether the studied biomarkers are correlated with serum MMP9.

In the present study, we attempted to explore the overall and stratified comparative differences in serum MMP9 levels and evaluate its potential clinical applicability for predicting AAA and TAA. Meanwhile, we intended to determine the relationship between serum MMP9 and other laboratory markers to discuss their possible interaction relevant for aortic aneurysm.

\section{Methods}

\section{Subjects}

This was a single center, case-control study. A total of 105 AAA patients, 79 TAA patients and 112 controls were recruited from the First Hospital of China Medical University between October 2016 and October 2017. Thoracoabdominal aortic aneurysms and AAA cases with extension to the iliac artery were not included in this study. The diagnosis of all patients was based on the computed tomography angiography (CTA). Exclusion criteria included the subjects accompanied by congenital genetic disorders, severe cardiovascular diseases, autoimmune diseases, severe organ failure, infectious diseases, malignant tumors, hematological system diseases, previous aortic surgery or received non-steroidal anti-inflammatory drugs or steroids. Demographic data, risk factors and laboratory parameters were obtained from clinical records. Hypertension was defined as having a systolic blood pressure (SBP) $\geq 140 \mathrm{mmHg}$ and/or having a diastolic blood pressure (DBP) $\geq 90 \mathrm{mmHg}$ and/or being under antihypertensive treatment. Diabetes was defined as fasting serum glucose (FPG) $\geq 7 \mathrm{mmol} / \mathrm{L}(126 \mathrm{mg} / \mathrm{dL}$ ) and (or) being on treatment for diabetes [12]. Hyperlipidemia was defined as serum total cholesterol (TC) $\geq 6.22 \mathrm{mmol} / \mathrm{L}(240 \mathrm{mg} / \mathrm{dL})$, or serum triglyceride (TG) $\geq 2.26 \mathrm{mmol} / \mathrm{L}$ ( $200 \mathrm{mg} / \mathrm{dL}$ ), or serum low-density lipoprotein cholesterol (LDL-C) $\geq 4.14 \mathrm{mmol} / \mathrm{L}(160 \mathrm{mg} / \mathrm{dL})$ [13]. For subjects with AAA or TAA, the maximal aortic diameter was assessed via CTA using the average of three measurements. This study was approved by the Ethics Committee of the First Hospital of China Medical University (Shenyang, China). Written informed consent was obtained from each subject.

\section{Detection of serum MMP9}

Blood samples were collected using standardized sterile tubes and centrifuged at $3500 \mathrm{r} / \mathrm{min}$ for $10 \mathrm{~min}$ at $4{ }^{\circ} \mathrm{C}$, and the serum was separated, and stored at $-80{ }^{\circ} \mathrm{C}$ until being assayed. Serum MMP9 levels were measured by enzyme-linked immunosorbent assay (ELISA) using MMP9 ELISA kits (Wuhan Boster Biotechnology Company, Wuhan, Hubei, China), according to the manufacturer's protocol.

\section{Statistical analysis}

All statistical analyses were performed using SPSS version 17.0 software. Continuous variables were reported as mean values and standard deviations, and categorical variables were represented as numbers and percentages. Differences among categories were evaluated using ANOVA, independent-sample t-test or $\chi^{2}$ test as appropriate. Spearman's rank correlation test was used to examine the associations of serum MMP9 levels with laboratory markers and maximal aortic diameter. Multiple logistic regression models were performed to determine the predictive value of serum MMP9 in AAA or TAA risk with the adjustment for the potential confounding factors. Receiver operating characteristic (ROC) curves and the area under the curve (AUC) were used to evaluate the diagnostic effects of serum MMP9 and to determine appropriate cut-off points. A two-sided $P<0.05$ was considered statistically significant.

\section{Results}

\section{Characteristics of the study subjects}

The detailed clinical characteristics of the cases and controls were described and compared in Table 1. Male members made up a larger proportion in three groups. AAA and TAA patients tended to have higher heart rate, leucocyte count and blood pressure compared with control subjects.

\section{Serum MMP9 levels between different groups in the total and stratified comparisons}

Serum MMP9 levels in control, AAA and TAA groups were $258.79 \pm 133.00, \quad 333.45 \pm 138.57$ and $385.17 \pm 109.23 \mathrm{ng} / \mathrm{ml}$, respectively. In the total comparison, there were significantly higher serum MMP9 levels in AAA and TAA groups than those in control 
Table 1 Clinical characteristics of the study subjects

\begin{tabular}{|c|c|c|c|}
\hline \multirow[t]{2}{*}{ Variables } & Control & \multirow{2}{*}{$\begin{array}{l}\text { AAA } \\
N=105\end{array}$} & \multirow{2}{*}{$\begin{array}{l}\text { TAA } \\
N=79\end{array}$} \\
\hline & $N=112$ & & \\
\hline Age, years & $62.32 \pm 11.23$ & $66.75 \pm 10.28^{\#}$ & $59.04 \pm 11.55^{* \dagger}$ \\
\hline Males, n (\%) & $83(74.1 \%)$ & $78(74.2 \%)$ & $57(72.2 \%)$ \\
\hline Height, cm & $167.70 \pm 7.20$ & $169.49 \pm 7.06$ & $169.61 \pm 7.10$ \\
\hline Weight, kg & $70.12 \pm 10.13$ & $66.40 \pm 12.36^{\#}$ & $73.38 \pm 11.80^{\dagger}$ \\
\hline Heart rate, bmp & $73.58 \pm 9.67$ & $79.70 \pm 13.58^{\#}$ & $79.92 \pm 15.65^{*}$ \\
\hline Leucocyte, $\times 10^{9} / \mathrm{L}$ & $5.82 \pm 1.77$ & $7.94 \pm 3.53^{\#}$ & $11.16 \pm 4.87^{*+}$ \\
\hline Thrombocyte, $\times 10^{9} / \mathrm{L}$ & $221.51 \pm 56.72$ & $218.65 \pm 81.38$ & $207.88 \pm 64.66$ \\
\hline $\mathrm{SBP}, \mathrm{mmHg}$ & $134.48 \pm 17.19$ & $141.79 \pm 19.56^{\#}$ & $152.69 \pm 28.98^{*+}$ \\
\hline $\mathrm{DBP}, \mathrm{mmHg}$ & $77.70 \pm 11.67$ & $80.65 \pm 12.22$ & $86.44 \pm 18.33^{*}$ \\
\hline $\mathrm{TC}, \mathrm{mmol} / \mathrm{L}$ & $4.90 \pm 0.87$ & $4.75 \pm 1.01$ & $4.41 \pm 0.91^{*+}$ \\
\hline $\mathrm{TG}, \mathrm{mmol} / \mathrm{L}$ & $1.53 \pm 0.88$ & $1.40 \pm 1.06$ & $1.35 \pm 0.90$ \\
\hline $\mathrm{LDL}-\mathrm{C}, \mathrm{mmol} / \mathrm{L}$ & $3.13 \pm 0.77$ & $3.15 \pm 0.91$ & $2.68 \pm 0.74^{* \dagger}$ \\
\hline $\mathrm{HDL}-\mathrm{C}, \mathrm{mmol} / \mathrm{L}$ & $1.32 \pm 0.34$ & $1.04 \pm 0.31^{\#}$ & $1.13 \pm 0.37^{*}$ \\
\hline $\mathrm{FPG}, \mathrm{mmol} / \mathrm{L}$ & $5.55 \pm 1.63$ & $5.74 \pm 1.56$ & $6.43 \pm 1.84^{* \dagger}$ \\
\hline$C R P, m g / L$ & - & $32.97 \pm 51.71$ & $64.95 \pm 47.47^{\dagger}$ \\
\hline Hcy, umol/L & - & $18.60 \pm 11.60$ & $18.52 \pm 12.77$ \\
\hline Cys-c, mg/L & - & $1.14 \pm 0.44$ & $1.40 \pm 0.76^{\dagger}$ \\
\hline Max. aortic diameter, $\mathrm{cm}$ & - & $5.64 \pm 1.61$ & $5.28 \pm 0.95$ \\
\hline
\end{tabular}

$A A A$ abdominal aortic aneurysm, TAA thoracic aortic aneurysm

$P^{\#}$ : AAA vs. Control, $P^{*}$ : TAA vs. Control, $P^{\dagger}$ : TAA vs. AAA

group, while TAA subjects had higher serum MMP9 levels when compared with AAA patients(all $P<0.05$ ).

Furthermore, we compared serum MMP9 levels between different groups stratified by age, gender, hypertension, diabetes or hyperlipidemia status and maximal aortic diameter, as shown in Table 2. The results showed that MMP9 levels were significantly increased from control to AAA to TAA group in the subjects aged $<65$ years. Compared to control group, AAA and TAA patients had increased MMP9 levels in either male or female, and this discrepancy was also found in hypertension status. Meanwhile, serum MMP9 levels in TAA patients were significantly higher than those in AAA subjects in male subgroup comparison. However, in the non-diabetes and non-hyperlipidemia status, MMP9 levels were obviously higher from control to AAA to TAA group (all $P<0.05$ ). When stratified by maximal aortic diameter, TAA patients tended to have higher MMP9 levels than AAA cases only in the subgroup with max. Aortic diameter $\geq 5.5 \mathrm{~cm}$.

Influence of age, gender, risk factors and maximal aortic diameter on serum MMP9

Table 2 also showed the comparison results in the individual group. We found that the $\geq 65$ years group had much higher serum MMP9 levels compared with the $<65$ years group in the control and AAA group. Serum MMP9 levels were significantly higher in subjects with hypertension than those without hypertension in each group (all $P<0.05$ ). However, there were no significant differences in the serum MMP9 levels between male and female, diabetes and non-diabetes, hyperlipidemia and non- hyperlipidemia, max. Aortic diameter $\geq 5.5 \mathrm{~cm}$ and $<5.5 \mathrm{~cm}$ groups.

\section{Correlation of serum MMP9 with laboratory biomarkers and maximal size of aneurysm}

We evaluated a possible correlation between MMP9 levels and CRP, Cys-c, Hcy and maximal aortic diameter. Serum MMP9 levels had a positive association with the concentration of $\operatorname{CRP}(r=0.330, P<0.001)$ and $\operatorname{Hcy}(r=0.199, P=0.033)$ (Fig. 1$)$. However, there was no significant relationship of MMP9 levels with Cys-c $(r=0.097, P=0.272)$ and maximal aortic diame$\operatorname{ter}(r=0.008, P=0.918)$.

\section{Predictive and diagnostic value of serum MMP9 for aortic} aneurysm

We further performed multiple logistic regressions to evaluate the risk prediction value of serum MMP9 for AAA and TAA under different adjustment models, as shown in Table 3 . When all potential confounding factors were adjusted, serum MMP9 was still significantly associated with AAA risk $(\mathrm{OR}=1.004$ per unit increase, $95 \% \mathrm{CI}$ 


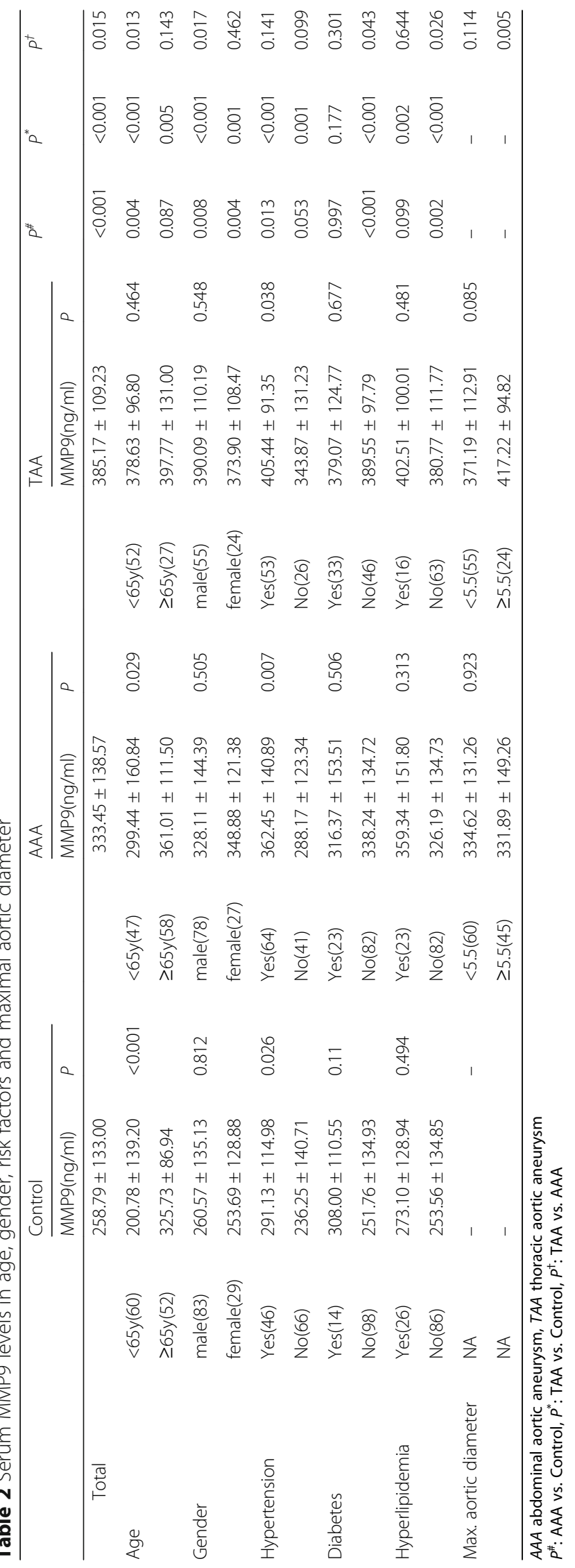



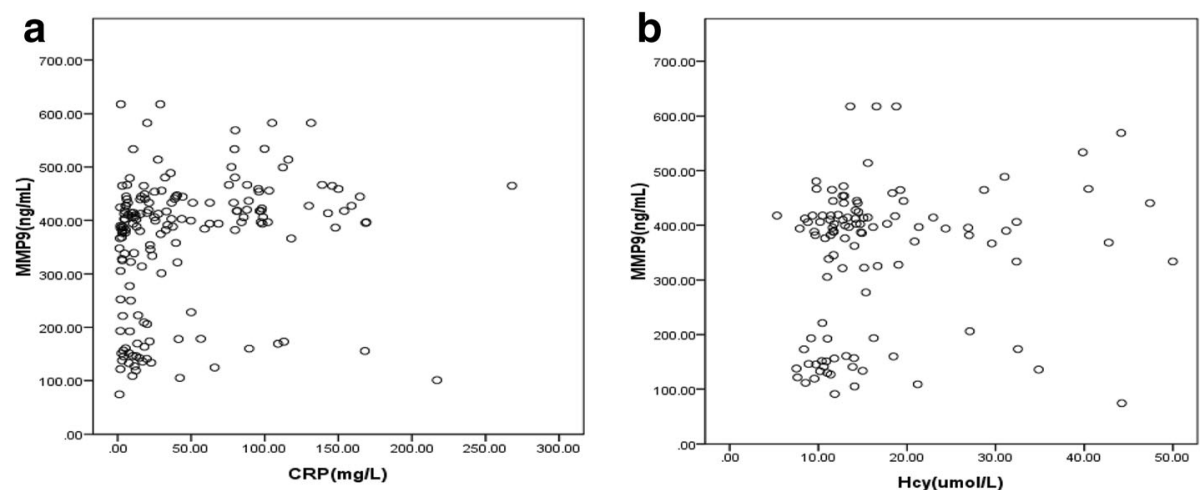

Fig. 1 Scatter plots for the association of serum MMP9 with CRP(a) and Hcy(b)

$=1.001-1.007, P=0.018)$ and TAA risk $(\mathrm{OR}=1.014$ per unit increase, $95 \% \mathrm{CI}=1.006-1.022, P<0.001)$.

In addition, the ROC curves of MMP9 levels for predicting AAA and TAA (Fig. 2). The ROC curve analysis illustrated that MMP9 levels had strong diagnostic value for TAA with the AUC of 0.83(95\% CI: $0.77-0.90$; $P<0.001)$ and an optimal cut-off point of $393.00 \mathrm{ng} / \mathrm{ml}$ associated with corresponding validity parameters of $70 \%$ sensitivity and $91 \%$ specificity. However, the AUC of MMP9 for predicting AAA was 0.69(95\% CI: $0.62-$ $0.76 ; P<0.001)$ and MMP9 $\geq 385.32 \mathrm{ng} / \mathrm{ml}$ had a sensitivity of $50 \%$ and a specificity of $88 \%$.

\section{Discussion}

Serum MMP9 levels represent the leakage of enzyme into the bloodstream during periods of matrix catabolism and its elevation may reflect a more active state of degeneration of the aortic wall. In the current study, our results suggested higher MMP9 levels in either AAA or TAA group than those in control group. Interestingly, we also found that TAA patients tended to have higher MMP9 levels than AAA subjects in the overall comparison, which might depend on their different embryological feature, wall mechanics and arterial hemodynamics [2]. Compared to the abdominal aorta, thoracic aorta has thicker aortic media
$[14,15]$ and a higher degree of wall shear stress [16, 17], which were possibly linked to higher MMP9 production for aneurysm formation.

In the subgroup comparisons stratified by age, gender, hypertension, diabetes and hyperlipidemia, we found that both AAA and TAA patients had higher MMP9 levels in the subjects aged $<65$ years, either male or female, and hypertensive status compared with controls. In addition, MMP9 levels showed to increase from control to AAA to TAA group in the non-diabetes and non-hyperlipidemia status. Hypertension is a well-known risk factor associated with aortic aneurysm. However, although diabetes and hyperlipidemia are recognized cardiovascular risk factors strongly associated with most acquired cardiovascular pathologies, they seem to be relatively weak risk factors for aortic aneurysm. Some studies indicated that the presence of diabetes had a reduced risk for aortic aneurysm, resulting from decreased MMPs production and activation in the aortic wall $[18,19]$. Recent research also found that diabetes inhibited experimental aortic aneurysm progression through reducing macrophage infiltration and medial elastolysis, and lowering serum glucose could diminish its protective effects [20, 21]. Hyperlipidemia and its associated effect on extracellular matrix have also

Table 3 Multiple logistic regression analysis of serum MMP9 levels for AAA and TAA risk

\begin{tabular}{|c|c|c|c|c|}
\hline Variables & AAA & & TAA & \\
\hline & $\mathrm{OR}(95 \% \mathrm{Cl})$ & $P$ & $\mathrm{OR}(95 \% \mathrm{Cl})$ & $P$ \\
\hline \multicolumn{5}{|l|}{ Model 1} \\
\hline MMP9, ng/mL & $1.004(1.002-1.006)$ & $<0.001$ & $1.010(1.007-1.013)$ & $<0.001$ \\
\hline \multicolumn{5}{|l|}{ Model 2} \\
\hline MMP9, ng/mL & $1.004(1.001-1.007)$ & 0.006 & $1.012(1.007-1.018)$ & $<0.001$ \\
\hline \multicolumn{5}{|l|}{ Model 3} \\
\hline MMP9, ng/mL & $1.004(1.001-1.007)$ & 0.018 & $1.014(1.006-1.022)$ & $<0.001$ \\
\hline
\end{tabular}

Model 1: age and gender were adjusted

Model 2: Model 1 plus height, weight, heart rate, leucocyte and thrombocyte

Model 3: Model 2 plus hypertension, diabetes and hyperlipidemia 

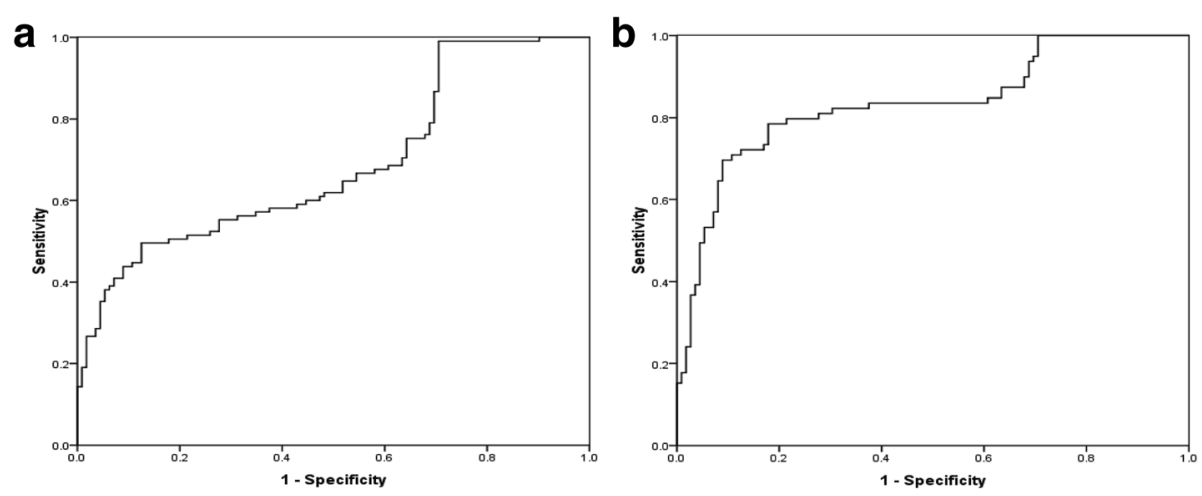

Fig. 2 ROC curve for serum MMP9 levels to predict AAA (a) and TAA (b)

been demonstrated to limit AAA formation in mice [21], and showed a negative association with AAA risk [22, 23]. These observations identified the protective role of diabetes and hyperlipidemia in aneurysm formation, which might partly account for our findings that the different MMP9 levels were obviously reflected in non-diabetic and non-hyperlipidemia subgroup analysis. Furthermore, we analyzed the effects of traditional cardiovascular risk factors on serum MMP9 levels. We found that subjects aged $\geq 65$ years had higher MMP9 levels than those aged $<65$ years. Additionally, hypertensive participants exhibited much higher serum MMP9 levels than non-hypertensive ones in each group.

Maximal aortic diameter, as a surrogate clinical marker of the growth rate, has been used to discuss its potential correlation with MMP9 levels [16]. MMP9 activity varied with aortic diameter in AAA, and its expression was reported to be elevated in aneurysms with a diameter of 5.0 to $6.9 \mathrm{~cm}$ [24]. Freestone et al. [25] indicated an increased activity of MMP9 in aneurysms with a diameter $\geq 5.5 \mathrm{~cm}$. On the contrary, neither Hovsepian nor Eugster had observed a significant correlation between serum MMP9 and aneurysm size of AAA [26, 27]. A recent study also suggested no relationship between serum MMP9 and TAA diameter [9]. In our study, serum MMP9 levels were not influenced by maximal aortic diameter in either AAA or TAA group, however, TAA patients were prone to have higher MMP9 levels than AAA subjects in aortic diameter $\geq 5.5 \mathrm{~cm}$ subgroup comparison.

Various systemic laboratory diagnostic biomarkers have been investigated and linked to the risk for aortic aneurysm or its outcomes, such as CRP, Hcy and Cys-c. CRP, a sensitive and non-specific inflammatory marker, has been recognized as an independent risk factor in the detection of vascular inflammation [4]. It has been considered to be associated with aortic aneurysm presence and progression $[28,29]$. Moreover, CRP can induce MMP9 production in human mononuclear cells in a concentration-dependent manner [30,31]. Hcy, a sulfur containing amino acid, has been suggested to play a key role in aortic aneurysm [32, 33]. Moreover, Hcy can produce marked vascular remodeling and elastolysis of the aortic wall by excessive MMPs production and activation $[10,34]$. Above evidence might explain our findings that serum MMP9 levels were positively correlated with circulating CRP and Hcy, which could partly reflect the fact that multiple biomarkers responded to aortic aneurysm related events. Although circulating Cys-c has been proved to be related to AAA and may favor proteolysis in the pathogenesis of AAA [35], we found no association of serum MMP9 with Cys-c.

To further assess the clinical application value of serum MMP9 in aortic aneurysm, we explored its predictive and diagnostic efficacy for identifying AAA or TAA. In multiple logistic analyses after adjusting the possible confounders, we demonstrated that serum MMP9 was an independent risk factor for the existence of AAA or TAA. With a low expected incidence, the focus for a biomarker should primarily be on specificity. Based on ROC curves, elevated MMP9 levels suggested a higher specificity than sensitivity in recognizing either AAA or TAA, which represented that serum MMP9 conferred a crucial role in safely ruling out aortic aneurysm. Moreover, AUCs, cut-off points for serum MMP9, and the sensitivity and specificity were much better with its use for predicting TAA than AAA. Therefore, serum MMP9 may be a valuable diagnostic biomarker for aortic aneurysm, especially for TAA.

Our study has some limitations. First, our sample size was small, and a more precise localization and detailed classification for TAA cases were lacking. Second, the serial measurements of MMP9 levels for evaluating dynamic changes were not investigated. Third, there were no complete records of potential confounders relevant to aortic aneurysm and MMP9 levels, such as the history of smoking and drinking. In addition, histological samples and MMP9 tissue expression were not investigated in this study. Further prospective studies based on larger 
scale cohorts will be needed to validate the clinical applicability of serum MMP9 on the identification of aortic aneurysm initiation and progression in the future practice.

\section{Conclusions}

Our results suggested that MMP9 levels were significantly increased in AAA and TAA group. Furthermore, we also found TAA patients tended to have higher levels of MMP9 than AAA subjects in the overall comparison, and in the non-diabetes, non-hyperlipidemia and aortic diameter $\geq 5.5 \mathrm{~cm}$ subgroup analysis. Moreover, MMP9 levels were affected by age and hypertension, and were positively associated with circulating CRP and Hcy. Multiple logistic analyses further suggested that serum MMP9 was an independent risk factor for aortic aneurysm. Serum MMP9 showed a high specificity for the diagnosis of either AAA or TAA. Therefore, MMP9 might be a useful biomarker with clinical predictive and diagnostic value for aortic aneurysm, especially for TAA.

\section{Abbreviations \\ AAA: Abdominal aortic aneurysm; AUC: Area under the curve; CRP: C-reactive protein; CTA: Computed tomography angiography; Cys-C: Cystatin C; DBP: Diastolic blood pressure; ELISA: Enzyme-linked immunosorbent assay; FPG: Fasting serum glucose; Hcy: Homocysteine; HDL-C: High-density lipoprotein cholesterol; LDL-C: Low-density lipoprotein cholesterol; MMP9: Matrix metalloproteinase-9; ROC: Receiver operating characteristic; SBP: Systolic blood pressure; TAA: Thoracic aortic aneurysm; TC: Total cholesterol; TG: Triglyceride}

\section{Acknowledgments}

The authors would like to thank Professor Yuan Yuan and Shi-jie Xin for their assistance for the coordination of this study.

\section{Funding}

This work is supported by grants from the Natural Science Foundation of Liaoning Province (Ref No. 2015020506).

\section{Availability of data and materials}

The datasets generated and/or analyzed during the current study are available from the corresponding author on reasonable request.

\section{Authors' contributions}

$T L$ performed the experiments, analyzed the data and drafted the manuscript. $\mathrm{BJ}$ and $\mathrm{XL}$ contributed to the clinical data and blood sample collection. HYS, XTL and JJ J participated in the experiments. JY was involved in supervisory role in study design. All authors have read and approved the final manuscript.

\section{Ethics approval and consent to participate}

This study was approved by the Ethics Committee of the First Hospital of China Medical University. And informed consent was obtained from each subject.

\section{Consent for publication}

Consent for publication was obtained from each subject.

\section{Competing interests}

All authors declare that they have no competing interests.

\section{Publisher's Note}

Springer Nature remains neutral with regard to jurisdictional claims in published maps and institutional affiliations.

\section{Author details}

'Department of Cardiovascular Ultrasound, the First Hospital of China Medical University, No.155 Nanjing Bei Street, Heping District, Shenyang 110001, China. ${ }^{2}$ Department of Vascular and Thyroid Surgery, the First Hospital of China Medical University, Shenyang 110001, China. ${ }^{3}$ Tumor Etiology and Screening Department of Cancer Institute and General Surgery, the First Hospital of China Medical University, Shenyang 110001, China.

Received: 5 December 2017 Accepted: 8 October 2018

Published online: 29 October 2018

\section{References}

1. Baumann F, Makaloski V, Diehm N. Aortic aneurysms and aortic dissection: epidemiology pathophysiology and diagnostics. Der Internist. 2013;54(5): 535-42.

2. Guo DC, Papke CL, He R, Milewicz DM. Pathogenesis of thoracic and abdominal aortic aneurysms. Ann N Y Acad Sci. 2006;1085:339-52.

3. Matsumoto Kl, Satoh K, Maniwa T, Tanaka T, Okunishi H, Oda T. Proteomic comparison between abdominal and thoracic aortic aneurysms. Int J Mol Med. 2014:33(4):1035-47.

4. Wen D, Zhou XL, Li JJ, Hui RT. Biomarkers in aortic dissection. Clin Chim Acta. 2011:412(9-10):688-95.

5. Grodin JL, Powell-Wiley TM, Ayers CR, Kumar DS, Rohatgi A, Khera A, McGuire DK, de Lemos JA, Das SR. Circulating levels of matrix metalloproteinase- 9 and abdominal aortic pathology: from the Dallas heart study. Vasc Med. 2011:16(5):339-45.

6. Takagi H, Manabe H, Kawai N, Goto SN, Umemoto T. Circulating matrix metalloproteinase-9 concentrations and abdominal aortic aneurysm presence: a meta-analysis. Interact Cardiovasc Thorac Surg. 2009;9(3):437-40.

7. Rabkin SW. Differential expression of MMP-2, MMP-9 and TIMP proteins in thoracic aortic aneurysm - comparison with and without bicuspid aortic valve: a meta-analysis. Vasa. 2014;43(6):433-42.

8. Zhang X, Wu D, Choi JC, Minard CG, Hou X, Coselli JS, Shen YH, LeMaire SA. Matrix metalloproteinase levels in chronic thoracic aortic dissection. J Surg Res. 2014;189(2):348-58.

9. Meffert P, Tscheuschler A, Beyersdorf F, Heilmann C, Kocher N, Uffelmann X, Discher $\mathrm{P}$, Rylski B, Siepe M, Kari FA. Characterization of serum matrix metalloproteinase 2/9 levels in patients with ascending aortic aneurysms. Interact Cardiovasc Thorac Surg. 2017:24(1):20-6.

10. Tsarouhas K, Tsitsimpikou C, Apostolakis S, Haliassos A, Tzardi M, Panagiotou M, Tsatsakis A, Spandidos DA. Homocysteine and metalloprotease-3 and -9 in patients with ascending aorta aneurysms. Thromb Res. 2011;128(5):e95-9.

11. Karapanagiotidis GT, Antonitsis P, Charokopos N, Foroulis CN, Anastasiadis K, Rouska E, Argiriadou H, Rammos K, Papakonstantinou C. Serum levels of matrix metalloproteinases $-1,-2,-3$ and -9 in thoracic aortic diseases and acute myocardial ischemia. J Cardiothorac Surg. 2009;4:59.

12. Li T, Chen S, Guo X, Yang J, Sun Y. Impact of hypertension with or without diabetes on left ventricular remodeling in rural Chinese population: a crosssectional study. BMC Cardiovasc Disord. 2017;17(1):206

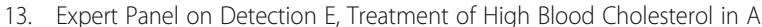
Executive summary of the third report of the National Cholesterol Education Program (NCEP) expert panel on detection, evaluation, and treatment of high blood cholesterol in adults (adult treatment panel III). Jama. 2001:285(19):2486-97.

14. Davis FM, Rateri DL, Daugherty A. Mechanisms of aortic aneurysm formation: translating preclinical studies into clinical therapies. Heart. 2014; 100(19):1498-505

15. Ito S, Akutsu K, Tamori Y, Sakamoto S, Yoshimuta T, Hashimoto H, Takeshita S. Differences in atherosclerotic profiles between patients with thoracic and abdominal aortic aneurysms. Am J Cardiol. 2008;101(5):696-9.

16. Astrand H, Ryden-Ahlgren A, Sundkvist G, Sandgren T, Lanne T. Reduced aortic wall stress in diabetes mellitus. Eur J Vasc Endovasc Surg. 2007;33(5): 592-8.

17. Castier Y, Brandes RP, Leseche G, Tedgui A, Lehoux S. p47phox-dependent NADPH oxidase regulates flow-induced vascular remodeling. Circ Res. 2005; 97(6):533-40.

18. Gertz SD, Gavish L, Mintz Y, Beeri R, Rubinstein C, Gavish LY, Berlatzky Y, Appelbaum L, Gilon D. Contradictory effects of hypercholesterolemia and diabetes mellitus on the progression of abdominal aortic aneurysm. Am 」 Cardiol. 2015;115(3):399-401. 
19. Golledge J, Karan M, Moran CS, Muller J, Clancy P, Dear AE, Norman PE. Reduced expansion rate of abdominal aortic aneurysms in patients with diabetes may be related to aberrant monocyte-matrix interactions. Eur Heart J. 2008;29(5):665-72.

20. Dua MM, Miyama N, Azuma J, Schultz GM, Sho M, Morser J, Dalman RL. Hyperglycemia modulates plasminogen activator inhibitor-1 expression and aortic diameter in experimental aortic aneurysm disease. Surgery. 2010; 148(2):429-35.

21. Miyama N, Dua MM, Yeung JJ, Schultz GM, Asagami T, Sho E, Sho M, Dalman RL. Hyperglycemia limits experimental aortic aneurysm progression. J Vasc Surg. 2010:52(4):975-83.

22. Chun KC, Teng KY, Chavez LA, Van Spyk EN, Samadzadeh KM, Carson JG, Lee ES. Risk factors associated with the diagnosis of abdominal aortic aneurysm in patients screened at a regional veterans affairs health care system. Ann Vasc Surg. 2014;28(1):87-92.

23. Golledge J, van Bockxmeer F, Jamrozik K, McCann M, Norman PE. Association between serum lipoproteins and abdominal aortic aneurysm. Am J Cardiol. 2010;105(10):1480-4.

24. McMillan WD, Tamarina NA, Cipollone M, Johnson DA, Parker MA, Pearce WH. Size matters: the relationship between MMP-9 expression and aortic diameter. Circulation. 1997;96(7):2228-32.

25. Freestone T, Turner RJ, Coady A, Higman DJ, Greenhalgh RM, Powell JT. Inflammation and matrix metalloproteinases in the enlarging abdominal aortic aneurysm. Arterioscler Thromb Vasc Biol. 1995;15(8):1145-51.

26. Eugster T, Huber A, Obeid T, Schwegler I, Gurke L, Stierli P. Aminoterminal propeptide of type III procollagen and matrix metalloproteinases-2 and -9 failed to serve as serum markers for abdominal aortic aneurysm. Eur I Vasc Endovasc Surg. 2005;29(4):378-82.

27. Hovsepian DM, Ziporin SJ, Sakurai MK, Lee JK, Curci JA, Thompson RW Elevated plasma levels of matrix metalloproteinase-9 in patients with abdominal aortic aneurysms: a circulating marker of degenerative aneurysm disease. J Vasc Interv Radiol. 2000;11(10):1345-52.

28. Golledge J, Tsao PS, Dalman RL, Norman PE. Circulating markers of abdominal aortic aneurysm presence and progression. Circulation. 2008; 118(23):2382-92

29. Hellenthal FA, Buurman WA, Wodzig WK, Schurink GW. Biomarkers of abdominal aortic aneurysm progression. Part 2: inflammation. Nat Rev Cardiol. 2009;6(8):543-52.

30. Nabata A, Kuroki M, Ueba H, Hashimoto S, Umemoto T, Wada H, Yasu T, Saito M, Momomura S, Kawakami M. C-reactive protein induces endothelial cell apoptosis and matrix metalloproteinase-9 production in human mononuclear cells: implications for the destabilization of atherosclerotic plaque. Atherosclerosis. 2008;196(1):129-35.

31. Singh U, Dasu MR, Yancey PG, Afify A, Devaraj S, Jialal I. Human C-reactive protein promotes oxidized low density lipoprotein uptake and matrix metalloproteinase-9 release in Wistar rats. J Lipid Res. 2008;49(5):1015-23.

32. Moroz P, Le MT, Norman PE. Homocysteine and abdominal aortic aneurysms. ANZ J Surg. 2007;77(5):329-32

33. Narayanan N, Tyagi N, Shah A, Pagni S, Tyagi SC. Hyperhomocysteinemia during aortic aneurysm a plausible role of epigenetics. Int J Physiol Pathophysiol Pharmacol. 2013;5(1):32-42.

34. Giusti B, Marcucci R, Lapini I, Sestini I, Lenti M, Yacoub M, Pepe G. Role of hyperhomocysteinemia in aortic disease. Cell Mol Biol. 2004;50(8):945-52.

35. Schulte S, Sun J, Libby P, Macfarlane L, Sun C, Lopez-llasaca M, Shi GP, Sukhova GK. Cystatin C deficiency promotes inflammation in angiotensin IIinduced abdominal aortic aneurisms in atherosclerotic mice. Am J Pathol. 2010;177(1):456-63.

Ready to submit your research? Choose BMC and benefit from:

- fast, convenient online submission

- thorough peer review by experienced researchers in your field

- rapid publication on acceptance

- support for research data, including large and complex data types

- gold Open Access which fosters wider collaboration and increased citations

- maximum visibility for your research: over $100 \mathrm{M}$ website views per year

At BMC, research is always in progress.

Learn more biomedcentral.com/submissions 